Research Paper

\title{
Amoebozoa Possess Lineage-Specific Globin Gene Repertoires Gained by Individual Horizontal Gene Transfers
}

\author{
Jasmin Dröge ${ }^{1}$, Dorota Buczek ${ }^{1,2}$, Yutaka Suzuki³, and Wojciech Makałowski1,3凶 \\ 1. Institute of Bioinformatics, Faculty of Medicine, University of Muenster, Niels Stensen Str. 14, 48149 Muenster, Germany \\ 2. Institute of Molecular Biology and Biotechnology, A. Mickiewicz University, Poznan, Poland \\ 3. Department of Medical Genomic Sciences, University of Tokyo, Tokyo, Japan
}

\begin{abstract}
$\bowtie$ Corresponding author: wojmak@uni-muenster.de
( ) Ivyspring International Publisher. This is an open-access article distributed under the terms of the Creative Commons License (http://creativecommons.org/ licenses/by-nc-nd/3.0/). Reproduction is permitted for personal, noncommercial use, provided that the article is in whole, unmodified, and properly cited.
\end{abstract}

Received: 2013.12.10; Accepted: 2014.03.24; Published: 2014.06.19

\begin{abstract}
The Amoebozoa represent a clade of unicellular amoeboid organisms that display a wide variety of lifestyles, including free-living and parasitic species. For example, the social amoeba Dictyostelium discoideum has the ability to aggregate into a multicellular fruiting body upon starvation, while the pathogenic amoeba Entamoeba histolytica is a parasite of humans. Globins are small heme proteins that are present in almost all extant organisms. Although several genomes of amoebozoan species have been sequenced, little is known about the phyletic distribution of globin genes within this phylum. Only two flavohemoglobins (FHbs) of $D$. discoideum have been reported and characterized previously while the genomes of Entamoeba species are apparently devoid of globin genes. We investigated eleven amoebozoan species for the presence of globin genes by genomic and phylogenetic in silico analyses. Additional FHb genes were identified in the genomes of four social amoebas and the true slime mold Physarum polycephalum. Moreover, a single-domain globin (SDFgb) of Hartmannella vermiformis, as well as two truncated hemoglobins (trHbs) of Acanthamoeba castellanii were identified. Phylogenetic evidence suggests that these globin genes were independently acquired via horizontal gene transfer from some ancestral bacteria. Furthermore, the phylogenetic tree of amoebozoan FHbs indicates that they do not share a common ancestry and that a transfer of FHbs from bacteria to amoeba occurred multiple times.
\end{abstract}

Key words: Amoebozoa, globin genes

\section{Introduction}

Globins (Gbs) are small heme proteins that have been found in all kingdoms of life in a wide range of different species [1-3]. Gbs are able to bind various gaseous ligands, such as oxygen and nitric oxide, and have diverse functions, e.g. in respiration and nitric oxide detoxification [4]. The globin superfamily can be divided into three lineages, namely the $\mathrm{S}, \mathrm{F}$, and $\mathrm{T}$ globins, which belong to two structural classes [5-7]. The members of the $\mathrm{F}$ and $\mathrm{S}$ lineages possess the typical globin fold, i.e. a 3-over-3 (3/3) a-helical fold consisting of seven or eight a-helices, designated A through $\mathrm{H}$ [8]. In contrast, the members of the $\mathrm{T}$ lineage exhibit a $2 / 2$ structure characterized by a shortened or completely deleted A helix, a missing D helix, and the substitution of the proximal F helix by a polypeptide segment [9]. The F globin family consists of flavohemoglobins (FHbs), FHb-like globins with $\mathrm{N}$ and C-terminal extensions, and related single-domain globins (SDFgbs). The chimeric $\mathrm{FHb}$ proteins possess an N-terminal globin domain and a C-terminal FADand $\mathrm{NAD}(\mathrm{P}) \mathrm{H}$-binding reductase domain. Increasing evidence indicates that $\mathrm{FHbs}$ protect bacteria and 
simple eukaryotes, including yeast, against the toxic effects of nitric oxide likely via the nitroxylation of oxygen [10-13]. The $\mathrm{S}$ globin family comprises chimeric globin-coupled sensors (GCSs), protoglobins (Pgbs), and related single-domain globins (SDSgbs). The GCSs can be further categorized as either aerotactic or gene regulating [14]. The globins of the $\mathrm{T}$ lineage were named truncated hemoglobins (trHbs) because of their shortened primary structure. Based on phylogenetic analyses and structural differences the trHbs can be further divided into three groups, i.e. group I (trHbN), II (trHbO), and III (trHbP) [15, 16]. Several distinct functions for the trHbs have been proposed, including nitric oxide detoxification, oxygen/nitric oxide sensing, ligand/substrate storage, etc. $[15,17]$.

A model of globin evolution has been suggested in which the three lineages descent from a single common ancestor that likely resembled an extant SDFgb [3, 7]. It is assumed that globins emerged only in bacteria [7]. Later, the eukaryote and archaeal $3 / 3$ and $2 / 2$ globin genes originated from horizontal gene transfers (HGT) of bacterial SDFgb and trHb genes, respectively [5-7]. This is supported by several studies that demonstrate that HGT events shaped the phyletic distribution of globin genes in plants, fungi, and unicellular eukaryotes $[3,5,6,16,18-24]$. Finally, it is known that HGT events played a major role in the evolution of various species [25, 26].

Although the evolution of globins has been intensively studied in several species from all kingdoms of life, only little is known about their origins and distribution in unicellular eukaryotes, such as the Amoebozoa [27]. The Amoebozoa are a phylum of amoeboid protozoa that move by the means of pseudopodia. They are closely related to Opisthokonts (Metazoa/fungi group) and can be divided into six monophyletic clades within two main groups, the Lobosea and the Conosea [28, 29]. They adopted different habitats and life styles, such as free-living unicellular amoeba, obligate parasitic amoeba, social amoeba, and true slime molds. The free-living Amoebozoa are common inhabitants of soils and water, where they represent one of the main predators of bacteria [30]. Acanthamoeba castellanii is the most frequently found amoeba in soil and plays an important role in a variety of environments. One of the best-known amoebas is the social amoeba Dictyostelium discoideum that has been studied for decades. $D$. discoideum is a solitary amoeba that can achieve multicellularity under starvation by aggregation and morphogenesis into a fruiting body [31]. Some amoebas are known to cause infectious diseases in humans, for instance the intestinal parasite Entamoeba histolytica which can induce amebic colitis and amebic liver abscess [32]. Likewise, also some members of the genus Acanthamoeba have been identified as origin of amebic infections in humans [33].

The presented study aims to define the globin gene repertoire of amoebozoan species. Eleven different species of the subphyla Conosa (infraphyla Mycetozoa, Archamoebae) and Lobosa (infraphyla Tubulinea, Acanthopodina) were scanned for the presence of globin genes. We show that the examined Amoebozoa possess lineage-specific globin gene repertoires, composed of either FHbs, SDFgbs, or trHbs that have been likely gained through multiple horizontal gene transfer events from ancestral bacteria.

\section{Methods}

\section{Identification of amoebozoan globin genes}

Initially, the two previously described FHbs of D. discoideum [34] were used to search the non-redundant protein database of NCBI for homologous amoebozoan globin proteins employing the BLASTp algorithm with default parameters [35]. The retrieved sequences and the globin sequences of two fungi, Schizophyllum commune and Saccharomyces cerevisiae, were used to search the genomes of A. castellanii, D. discoideum, D. fasciculatum, D. purpureum, E. dispar, E. histolytica, E. invadens, E. moshkovskii, P. polycephalum and Polysphondylium pallidum for the presence of additional globin genes. This was done by a tBLASTn search applying varying parameters: e-value 0.1, 0.001, matrix: BLOSUM62, BLOSUM45, soft masking of query sequence. Likewise, a tBLASTn search was conducted against TBestDB [36]. Additionally, the transcriptome of $A$. castellanii was analyzed for the presence of globin sequences (Buczek et al., unpublished data). Subsequently, the similarity search was repeated with the newly found sequences. The intron/exon structures of the novel genes were manually annotated, guided by tBLASTn results, GENSCAN [37], and NetGene2 predictions [38, 39]. Intron positions with respect to the coding sequences were reported with reference to the secondary structure of the protein. This was done by aligning the amoebozoan globin proteins to myoglobin of the sperm whale (UniProtKB: P02185.2). FUGUE [40] and SMART [41] were used to validate the annotated sequences as globin genes. A table of sequences used in the subsequent analyses is provided in the supplementary materials (Table S1). All genes used in the consecutive analyses were labeled with first two letters of the binary species name and a suffix in cases multiple copies are present in a single genome.

Shared synteny is a reliable criterion to prove orthology between genomic segments and to trace back the evolution of those segments. The neighbor- 
ing genes of the FHbs from the social amoebas D. discoideum, D. purpureum, D. fasciculatum, and P. pallidum were identified using the genome browser and the tBLASTn tool provided by dictyBase [42, 43]. Orthologous genes were defined as reciprocal best hits (RBHs) via tBLASTn and BLASTp searches. Additionally, the direct neighbors of the trHbs of A. castellanii were determined via GENSCAN and subsequent BLASTp searches. We did not conduct a synteny analysis of the $\mathrm{FHb}$ genes of $P$. polycephalum due to the highly fragmented nature of the current genome assembly.

\section{Function inference analyses}

Several bacterial and metazoan globins are known to associate with the cell membrane [44-50]. To determine if the amoebozoan globins are potentially linked to the membrane, posttranslational modifications and transmembrane domains were predicted. The Myristoylator [51] and CSS-Palm 3.0 [52] servers were used to predict myristoylation and palmitoylation sites, respectively. The identification of transmembrane regions was done with TMpred [53] and TMHMM [54].

The secondary and tertiary structures of the amoebozoan globin proteins were modeled to further support inference of their functionality. Modeling was done using Swiss-Model and SwissPDBViewer 4.04 $[55,56]$, as described in [57]. Percent identity values of query and template sequences and e-values of the PSI-BLAST searches are provided in the supplementary materials (Table S2).

\section{Phylogenetic analysis}

In a genomic survey from 2009 among the three kingdoms of life more than 550, 253, and 668 globins of the F, S, and T lineages, respectively, were identified [3]. With ongoing sequence projects these numbers have further increased. Thus, the selection of a representative set of globin sequences for phylogenetic analyses is a challenging task. To receive a set of globin sequences that likely provide an insight into the evolution of amoebozoan globin proteins, the following steps were conducted. First, homologous globin proteins closely related to the amoebozoan globins were identified conducting BLASTp searches against the non-redundant protein database of NCBI. Thereby, for each amoebozoan globin the closest relative in bacteria, plants, fungi, archaea, and in eukaryotes other than plants, fungi, and Amoebozoa, was identified. This search was iterated for each received globin protein, till no new sequences could be added to the data set. Next, the sequence identity and similarity values of the received sequences were analyzed via MatGat [58]. Subsequently, redundant sequences were removed from the data set, e.g. highly similar globins from different subspecies. Based on ubiquitous clustering with high statistical support and short branches, sequences with identity equal or higher than 85 percent were considered as redundant and consequently only one from a given cluster was used for the phylogenetic inference.

Next, the sequences were divided into two data sets, one comprising FHbs (66 sequences), the other one trHbs (76). To identify overrepresented groups, i.e. clades containing several closely related species that do not provide any information on the origins and relationships of amoebozoan globins, a neighbor-joining tree was created employing PHYLIP 3.69 with default settings and 1,000 bootstrap replications [59]. This reduced the $\mathrm{FHb}$ and trHb data sets to 38 and 27 sequences, respectively. Multiple sequence alignments were conducted using MUSCLE 3.8.31 [60], MUSCLE 4.0 (preliminary, experimental version), the L-INS-i, G-INS-i and FFT-NS-i strategies of MAFFT [61, 62], and COBALT [63]. The best scoring alignment was chosen based on MUMSA scores [64]. The program packages RAxML 7.0.4 [65, 66] and MrBayes 3.1.2 [67, 68] were used for phylogenetic tree reconstructions. The best-fitting model of amino acid substitution was selected by the analysis of the alignment with ProtTest3 [69]. Phylogenetic analyses of the $\mathrm{FHb}$ and $\mathrm{trHb}$ data sets were based on the WAG [70] model of amino acid evolution, assuming gamma-distributed rate variation among sites. Maximum likelihood analyses were performed using the rapid bootstrapping RAxML algorithm with 1,000 bootstrap replications. The Bayesian interference was conducted using MrBayes 3.1.2. Metropolis-coupled Markov chain Monte Carlo sampling was performed with one cold and three heated chains that were run for $5,000,000$ generations in two independent runs. The trees were sampled every 1,000 th and 500th generation in the $\mathrm{FHb}$ and $\mathrm{trHb}$ analysis, respectively, and the 'burn in' was set to $25 \%$. Convergence of the runs was verified by assessing the average standard deviation of split frequencies, which reached values of 0.006193 and 0.001289 for the trHb and $\mathrm{FHb}$ data sets, respectively. Additionally, the parameters of the Bayesian inference were analyzed using Tracer [71]. For the calculation of the Bayesian trees, the CIPRES Science Gateway V.3.1 was used [72], while the phylogenetic trees were visualized with iTOL $[73,74]$.

CONSEL was used to test phylogenetic hypotheses [75]. The site-likelihoods for each tested tree topology were calculated applying TREE-PUZZLE [76]. Subsequently, the approximately unbiased (AU) test [77] was performed using CONSEL with default parameters. 
Table I. Genomic localization, gene length, number of exons, and protein length of annotated amoebozoan globin genes.

\begin{tabular}{|c|c|c|c|c|c|c|c|}
\hline Species & Globin type & Location & Coordinates & Strand & Gene length & Number of exons & Protein length \\
\hline Dictyostelium discoideum & $\mathrm{FHbA}$ & chromosome 6 & $1,649,565$ to $1,650,758$ & - & $1194 \mathrm{bp}$ & 1 & 397 \\
\hline Dictyostelium discoideum & $\mathrm{FHbB}$ & chromosome 6 & $1,651,520$ to $1,652,908$ & - & $1389 \mathrm{bp}$ & 2 & 423 \\
\hline Dictyostelium purpureum & $\mathrm{FHbA}$ & scaffold_485 & 14,681 to 16,277 & + & $1597 \mathrm{bp}$ & 3 & 392 \\
\hline Dictyostelium purpureum & $\mathrm{FHbB}$ & scaffold_530 & 9,247 to 10,772 & + & $1526 \mathrm{bp}$ & 1 & 423 \\
\hline Dictyostelium fasciculatum & $\mathrm{FHb}$ & DFA1501812 & $1,760,310$ to $1,763,147$ & - & $2837 \mathrm{bp}$ & 2 & 400 \\
\hline Polysphondylium pallidum & $\mathrm{FHb}$ & PPA1277996 & $2,450,684$ to $2,451,898$ & + & $1215 \mathrm{bp}$ & 1 & 404 \\
\hline Physarum polycephalum & $\mathrm{FHb}-1$ & contigs 10755,8539 & $\mathrm{~N} / \mathrm{D}$ & $\mathrm{N} / \mathrm{D}$ & $\mathrm{N} / \mathrm{D}$ & 71 & $375^{2}$ \\
\hline Physarum polycephalum & $\mathrm{FHb}-2$ & contigs 8539,3993 & $\mathrm{~N} / \mathrm{D}$ & $\mathrm{N} / \mathrm{D}$ & $\mathrm{N} / \mathrm{D}$ & 71 & $374^{2}$ \\
\hline Physarum polycephalum & $\mathrm{FHb}-3$ & contigs 8539,3993 & $\mathrm{~N} / \mathrm{D}$ & $\mathrm{N} / \mathrm{D}$ & $\mathrm{N} / \mathrm{D}$ & 9 & 376 \\
\hline Hartmannella vermiformis & SDFgb & $\mathrm{N} / \mathrm{D}$ & $\mathrm{N} / \mathrm{D}$ & $\mathrm{N} / \mathrm{D}$ & $\mathrm{N} / \mathrm{D}$ & $\mathrm{N} / \mathrm{D}$ & 159 \\
\hline Acanthamoeba castellanii & $\operatorname{trHbN}$ & GL877269 & 354,379 to 354,915 & - & 537 bp & 3 & 179 \\
\hline Acanthamoeba castellanii & $\operatorname{trHbO}$ & GL877210 & 157,074 to 157,782 & + & $709 \mathrm{bp}$ & 1 & 201 \\
\hline
\end{tabular}

1likely exons missing

estimated length

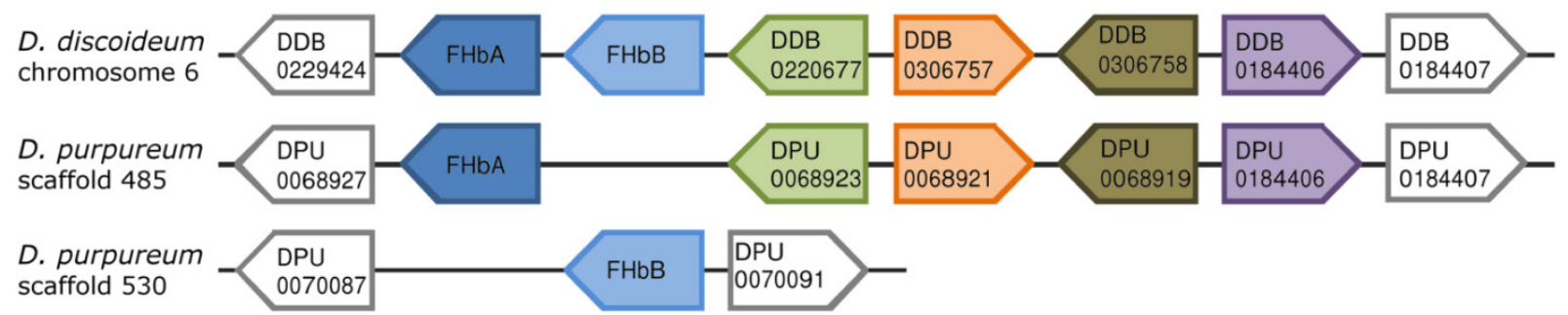

Figure I. Comparison of the genomic neighborhood of the FHb genes from $D$. discoideum and $D$. purpureum. The direct neighboring genes of the FHbA and FHbB genes of $D$. discoideum and $D$. purpureum are shown. The directions of the boxes indicate the genomic orientations of the genes, i.e. a box directed to the right equates the plus strand, directed to the left equates the minus strand. Boxes with the same color represent orthologs, while grey boxes indicate that those genes do not possess an ortholog in this genomic location. For each gene either the gene symbol or the accession number provided by dictyBase is given. The FHbs of $D$. discoideum are located on chromosome 6 in a head-to-tail orientation. In contrast, the FHbs of D. purpureum are lying on two different scaffolds. The FHbA genes of the two amoebas lie in a short conserved syntenic block.

\section{Results}

\section{Identification of amoebozoan globin genes}

The previously characterized globins from $D$. discoideum were used to search the protein database of NCBI for additional amoebozoan globin proteins. Two FHbs of D. purpureum, an FHb of D. fasciculatum and an $\mathrm{FHb}$ of $P$. pallidum were identified. Additionally, an SDFgb of Hartmannella vermiformis was found in the EST database TBestDB. The obtained sequences were used to search against available amoebozoan genomes and the transcriptome of A. castellanii for further globin genes. As already reported, no globins are present in the genomes of Entamoeba parasites [5]. The slime mold P. polycephalum seems to possess three $\mathrm{FHb}$ genes. Although we could not detect any $\mathrm{FHb}$ genes, two putative trHb genes were found in the genome of A. castellanii. Table 1 summarizes detailed information of the analyzed globin genes.

\section{The Flavohemoglobins of social amoebas and the slime mold $\mathbf{P}$. polycephalum}

The FHb genes of D. discoideum (DidiA and Did$i B)$ are located next to each other on chromosome 6 in a head-to-tail orientation (Figure 1) [34]. DidiA is a single exon gene while DidiB contains two coding exons interrupted by an intron at position H2.1, i.e. between the first and second base of codon two in globin helix $\mathrm{H}$, of $117 \mathrm{bp}$ lengths (Figure 2). In contrast, the $\mathrm{FHb}$ genes of D. purpureum (DipuA, DipuB) are present on two different scaffolds. The DipuA gene lies on scaffold_485 and is disrupted by two introns at positions E1.1 and H9.1 of 74 and 78 bp lengths (Figure 2). The DipuB gene is a single exon gene, located on scaffold_530. Interestingly, the FHbA genes of both species lie at the $5^{\prime}$ end of a short genomic block of conserved synteny (Figure 1). A total of five genes are conserved in order and orientation between the two Dictyostelium species. The shared synteny indicates that the $\mathrm{FHbA}$ genes are orthologs, despite their different intron/exon structure. 


1gvh
Ecco
DipuA
DipuB
DidiA
DidiB
Difa
Popa
Phpo1
Phpo2
Phpo3
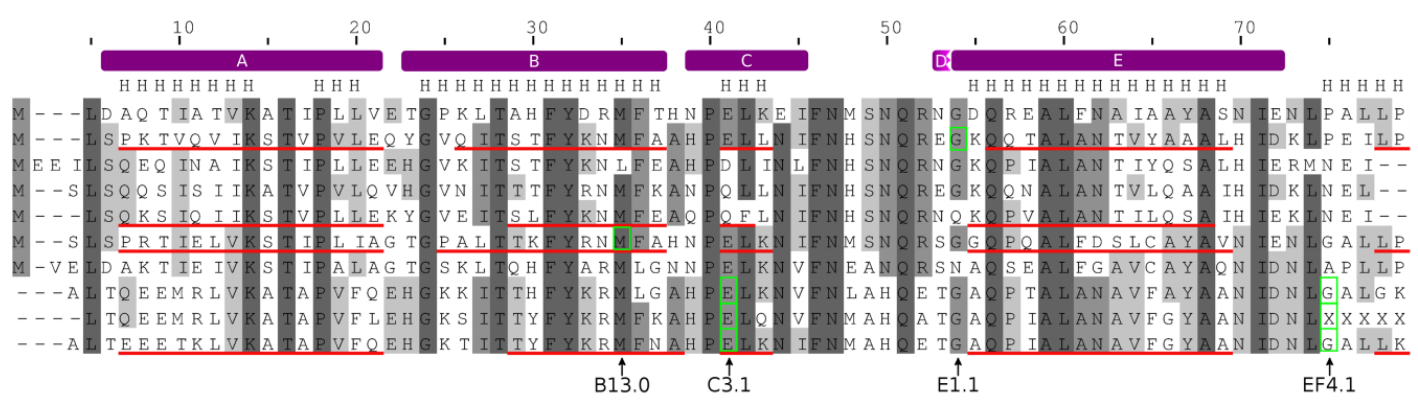

1gvh
Ecco
DipuA
DipuB
DidiA
DidiB
Difa
Popa
Phpo1
Phpo2
Phpo3

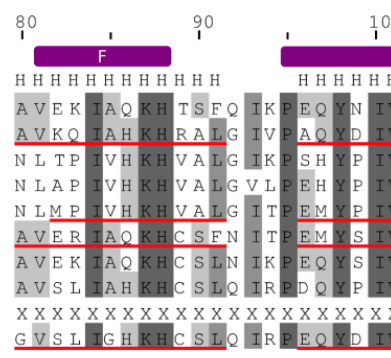

1gvh
Ecco
DipuA
DipuB
DidiA
DidiB
Difa
Popa
Phpo1
Phpo2
Phpo3

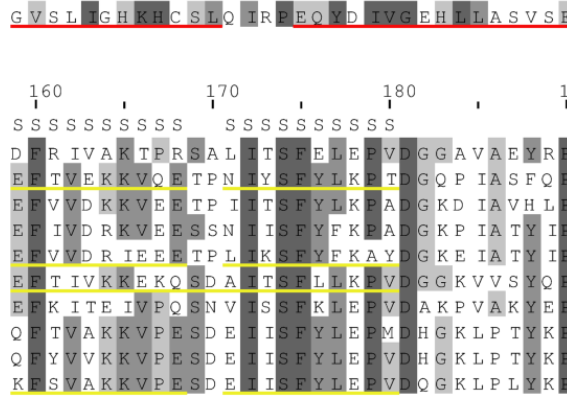

120

EF4.1

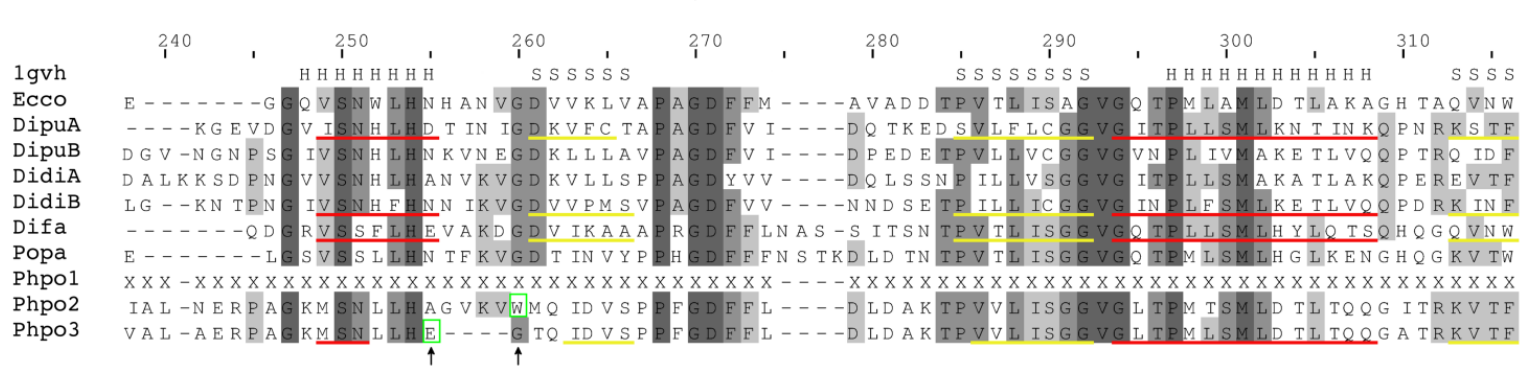

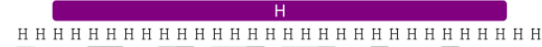

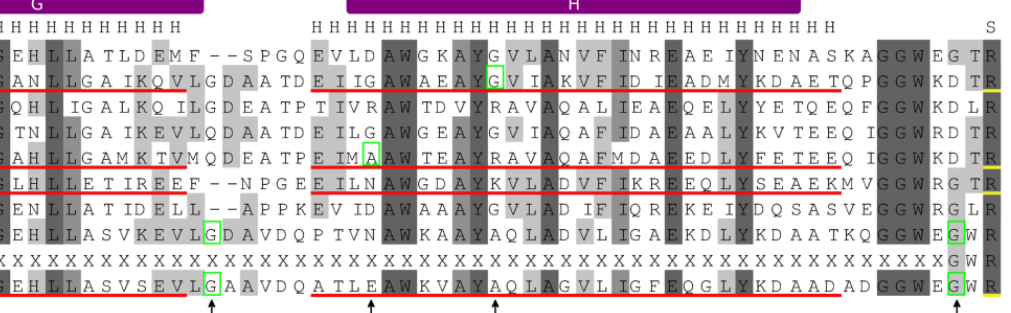

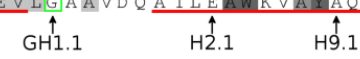

$190 \quad 200$

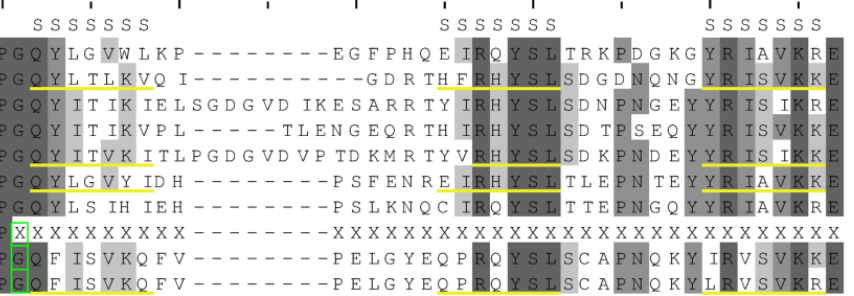

$\uparrow$

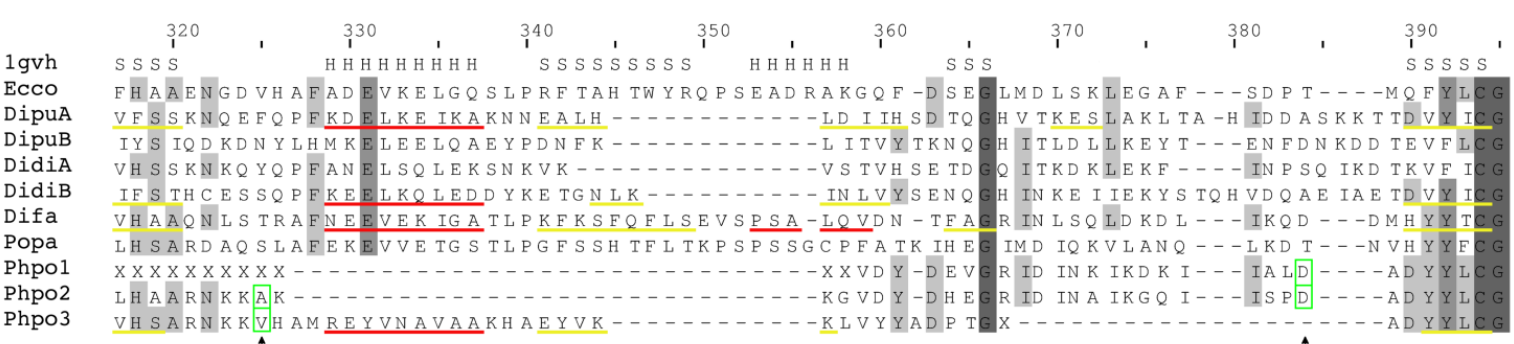

1gvh
Ecco
DipuA
DipuB
DidiA
DidiB
Difa
Popa
Phpo1
Phpo2
Phpo3

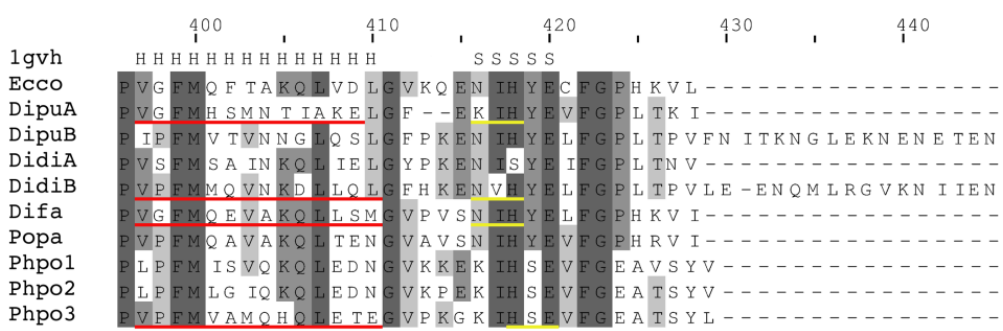

Figure 2. Alignment of amoebozoan FHbs to the Hmp protein of Escherichia coli. The alignment was created with MUSCLE. Conserved residues are shaded in different levels of grey. Residues that are conserved in all sequences are in dark grey. The secondary structure of the Hmp protein is given above the alignment (PDB: Igvh). Predicted $\alpha$-helices and $\beta$-strands are indicated as red and yellow lines, respectively, below the corresponding sequences. The positions of the introns are marked with green boxes and by arrows below the alignment. The topological positions of the introns as compared to sperm whale Mb are indicated below the alignment. The helix structure of the sperm whale myoglobin was superimposed on the alignment and indicated by violet bars above the alignment. 
In contrast, $D$. fasciculatum and P. pallidum possess only single $\mathrm{FHb}$ genes, which were found on supercontigs DFA1501812 and PPA1277996, respectively. The $\mathrm{FHb}$ of P. pallidum (Popa) is a single exon gene while the $\mathrm{FHb}$ of $D$. fasciculatum (Difa) likely consists of two coding exons, which are separated by an intron of approximately $1.7 \mathrm{~kb}$. The first exon codes for the first 32 amino acids of the globin domain. Although the sequence is highly conserved to other amoebozoan FHbs and comprises the A and B helix of the globin domain, no canonical splice sites were found. The current annotation places the intron at position B13.0 (Figure 2). No synteny conservation among the FHbs of $D$. fasciculatum and P. pallidum and to the other Dictyostelium species was observed.

The true slime mold $P$. polycephalum seems to possess three FHb genes (Phpo-1, Phpo-2, and Phpo-3), which span three potentially overlapping contigs (10755, 8539, and 3993). Thus, they are likely located next to each other in head-to-tail orientations in the genome. However, due to the highly fragmented nature of the current assembly, parts of the $\mathrm{FHb}$ genes are still missing. All globins are incomplete at their $\mathrm{N}$-terminal ends. Additionally, in Phpo-1 a large part of the flavin-containing oxidoreductase domain of about 125 amino acids is absent while in Phpo-2 half of the globin domain (helices $\mathrm{F}$ to $\mathrm{H}$ ) is missing. Nevertheless, it is highly likely that the missing exons lie in the still undetermined regions of the assembly. The Phpo-3 gene consists of nine exons and seems to be almost complete. However, the predicted splice sites of the last intron would result in two exons in different reading phase, which would consequently lead to a frame shift. Since the acceptor splice site is conserved among the Phpo genes, we assume that the second to last exon of Phpo-3 is incomplete at its 3' end. Three of the eight introns of Phpo-3 are lying in the globin domain at positions C3.1, EF4.1, and GH1.1 (Figure 2). The partial Phpo-1 and Phpo-2 genes each consist of seven exons interrupted by introns at the same positions as Phpo-3, except for introns four and six of Phpo-2 and Phpo-3, respectively. However, the position of the intron may change once undetermined regions are resolved.

The modeling of the tertiary structure of DidiB, DipuA, Difa, and Phpo-3 revealed that these globins can most likely adopt the typical globin fold. Nevertheless, as in the case of the hmp protein of Escherichia coli, the D helix seems to be absent [78]. All globins contain the highly conserved residues of the heme pocket, such as TyrB10, PheCD1, and HisF8 (proximal histidine). Moreover, also residues known to be responsible for FAD binding, e.g. Tyr206, Ser207, Phe390, Gly391 of E. coli hmp, are conserved among amoebozoan FHbs (Figure 2). Thus, we conclude that the amoebozoan FHbs likely represent functional proteins. The $\mathrm{FHb}$ proteins show no characteristics of membrane-bound proteins, and thus a membrane-association can be ruled out.

\section{Phylogeny of the amoebozoan Flavohemoglobins}

Phylogenetic trees of the amoebozoan FHbs and their closest relatives in the different kingdoms of life were reconstructed applying maximum likelihood and Bayesian interference algorithms. The MUSCLE 4.0 alignment (highest MUMSA score) was used for tree reconstruction and model selection. Both tree-building methods resulted in the same tree topology. Since no proper outgroup exists for our data set unrooted trees are presented. Figure 3 shows the maximum likelihood tree with superimposed bootstrap support and posterior probability values. Four highly supported clades can be identified of which three contain amoebozoan $\mathrm{FHb}$ proteins. The clade 1 comprises the FHbs of $D$. discoideum and D. purpureum, bacterial FHbs of some Firmicutes (Ocih, Brbr, Bame, Maca) and one $\gamma$-Proteobacterium (Pamu) as well as three fungal FHbs. Clade 2 encompasses the FHbs from P. pallidum and D. fasciculatum as well as the $\mathrm{FHb}$ of Gardia lamblia and $\mathrm{FHbs}$ of some $\gamma$-Proteobacteria (Enba, Encl, Vifi). The globins of $P$. polycephalum cluster together in clade 4 with $\mathrm{FHbs}$ from $\alpha$ - and $\beta$-Proteobacteria (Coin, Acar, Bope, Buok), one Cytophagium (Dyfe) and three fungal FHbs. This clade is sister to a group of fungal $\mathrm{FHb}$ proteins (clade 3 ). The $\mathrm{FHb}$ of $P$. sojae (Phso) seems to be unrelated to the other included FHbs.

\section{The truncated hemoglobins of $A$. castellanii}

We identified and annotated two trHbs of $A$. castellanii that were found in the genome as well as in the transcriptomic sequence data. The trHbs, named AccaN and AccaO, are located on the whole genome shotgun (wgs) contigs GL877269 and GL877210, respectively. Strikingly, the last four nucleotides of the AccaO mRNA, derived from the transcriptomic data, do not align to the genome. Moreover, the coding sequence (CDS) of the predicted genomic gene is 57 nucleotides longer than the CDS of the transcriptomic mRNA. The C-termini of both translated peptides do not show any significant similarities to other known proteins. We were not able to determine which sequence represents the true $A c c a O$ gene. We decided to use the transcriptomic data in the subsequent phylogenetic analyses.

In contrast to $A c c a N$, which is a single exon gene, AccaO contains two introns each $98 \mathrm{bp}$ long at positions B15.1 and G4.0 (Figure 4). To check for similarities in the genomic localization of the trHbs, the direct 
neighboring genes of $A c c a N$ and $A c c a O$ were determined. tBLASTn searches revealed that the upstream and downstream neighboring genes of AccaN are similar to an oxidoreductase and to a serine/threonine kinase, respectively, while the direct neighbors of $A c c a O$ resemble proteins with an mscl domain and an N-acetylglucosamine-1-phosphodiester alpha-4-acetylglucosaminidase.

Similarly to the $\mathrm{FHb}$ case, the tertiary structure of the trHbs has been predicted as described in the method section. Both globins are able to adopt the typical fold of truncated globins, including a short- ened A helix and absence of the D helix [9]. Furthermore, conserved residues of group I and II trHbs are present, such as the conserved glycine motifs, the Phe-Tyr pair at B9-10 and the proximal histidine (HisF8) (Figure 4). Thus, both globins likely represent functional proteins.

Interestingly, the AccaN protein may possess a transmembrane domain at its C-terminus (amino acids 159 - 176) as predicted by TMpred and TMHMM. Furthermore, CSS-Palm 3.0 predicted a potential palmitoylation site at Cys133. These findings indicate that AccaN may be a membrane-bound globin.

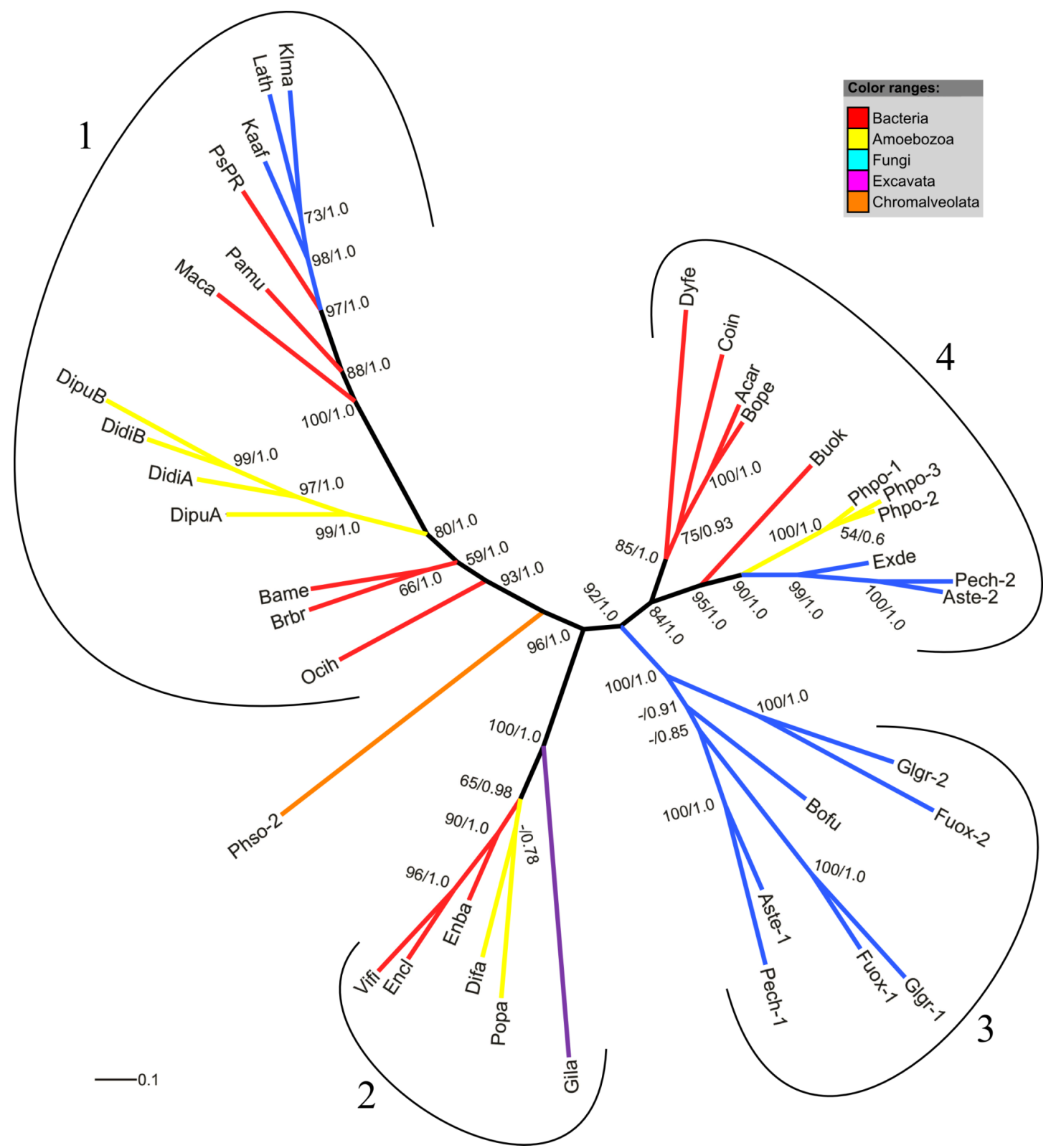

Figure 3. Radial maximum likelihood tree of FHb proteins. The colors of branches correspond to the taxonomic classification of the used sequences. Bootstrap support (bs) and posterior probability (PP) values equal or greater than $50 \%$ are given (bs/pP). The FHb proteins cluster in four highly supported clades (I-4). For a description of used abbreviations please refer to Supplementary Material: table SI. 


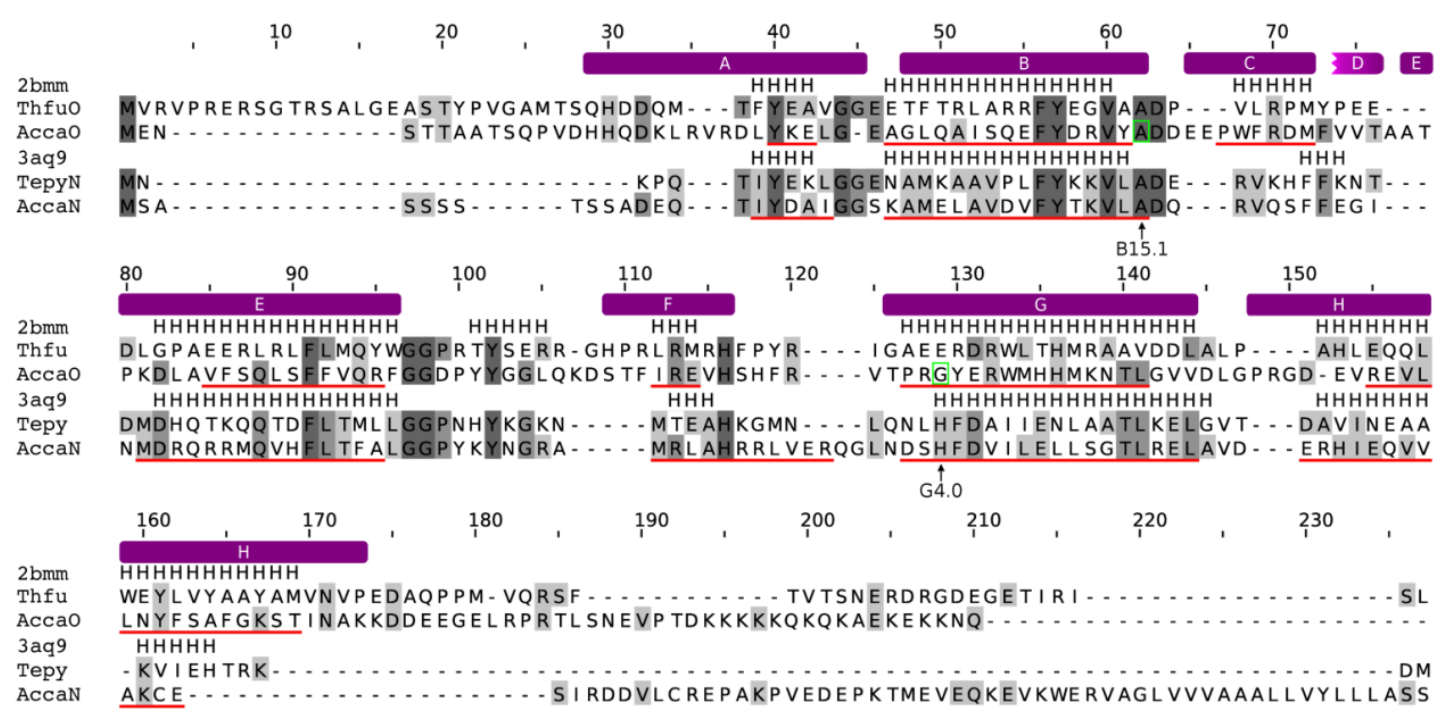

$\begin{array}{ll}2 \text { bmm } & \\ \text { Thfu } & \text { R - } \\ \text { AccaO } & - \\ \text { 3aq9 } & \\ \text { Tepy } & \text { LGK } \\ \text { AcCaN } & \text { Q - }\end{array}$

Figure 4. Alignment of the trHbs of A. castellanii to trHbN of Tetrahymena pyriformis and trHbO of Thermobifida fusca. The alignment was created with MUSCLE. Conserved residues are shaded in different levels of grey. Residues that are conserved in all sequences are in dark grey. The secondary structure of the trHb proteins of $T$. pyriformis (PDB: 3aq9) and T. fusca (PDB: $2 \mathrm{bmm}$ ) are given. Predicted $\alpha$-helices are indicated as red lines, below the corresponding sequences. The positions of the introns are marked with green boxes and by arrows below the alignment. The topological positions of the introns as compared to sperm whale Mb are indicated below the alignment. The helix structure of the sperm whale myoglobin was superimposed on the alignment and indicated by violet bars above the alignment.

\section{Phylogeny of the trHbs of A. castellanii}

Akin to the analysis of FHbs, phylogenetic trees of the trHbs of $A$. castellanii and its closest relatives were reconstructed. Here, the FFT-NS-i strategy of MAFFT produced the highest scoring MUMSA alignment. It is assumed that group I and group III globins likely represent the products of a duplication event of an ancestral group II gene [16]. The maximum likelihood and Bayesian interference analyses resulted in slightly different trees. However, clustering of the major clades was recovered in all analyses (Figure 5, Supplementary Material: Figure S1). Figure 5 shows the Maximum likelihood tree with superimposed bootstrap support values and posterior probability values of the Bayesian analysis. The trHbs cluster in accordance to their classifications in three monophyletic groups (I, II, III) (Figure 5). AccaO is positioned between the clades consisting of group II and group I/III globins while AccaN clusters together with a trHb of the castor oil plant (RicoN) and a putative $\mathrm{trHb}$ of a fungus (BadeN) in the clade comprising group I globins. Although, the clustering of AccaN with RicoN and BadeN is not well supported, it was recovered in all analyses.

\section{The single-domain SDFgb of Hartmannella vermiformis}

We identified an EST sequence of $H$. vermiformis in the TBestDB that shares high sequence similarity to F globin genes and most likely represents an SDFgb. Our analysis indicates that the EST sequence contains the complete CDS. The presence of a single globin domain in the translated peptide was verified via SMART. As for the FHbs, a membrane association of the SDFgb can be ruled out. BLASTp searches against the protein database of NCBI revealed that the closest relatives of the globin of $H$. vermiformis are bacterial SDFgbs and FHbs. Our phylogenetic trees further support this finding. In a tree comprising the different types of single-domain globins (SDFgbs, SDSgbs, Pgbs) the SDFgb of H. vermiformis (Have) clusters with two bacterial SDFgb proteins (Figure 6).

\section{Discussion}

\section{Evolution of amoebozoan globin genes}

Figure 7 summarizes our findings in the phylogenetic connects. The identification and characterization of amoebozoan globin genes revealed that two of the three major globin lineages are present in Amoebozoa, represented by FHbs, SDFgbs, and trHbs. The absence of members of the $S$ globin family is not surprising given that GCSs seem to be completely missing in eukaryotes and that SDSgbs have so far only been described in some bacteria, archaea, and fungi $[5,7]$. All analyzed species of the infraphylum Mycetozoa possess at least one $\mathrm{FHb}$ gene, while trHb genes 
and an SDFgb gene were only found in A. castellanii (infraphylum Acanthopodina) and H. vermiformis (infraphylum Tubulinea). These findings hint at line- age-specific adaptations of the globin gene repertoires although further research must be done to corroborate this belief.

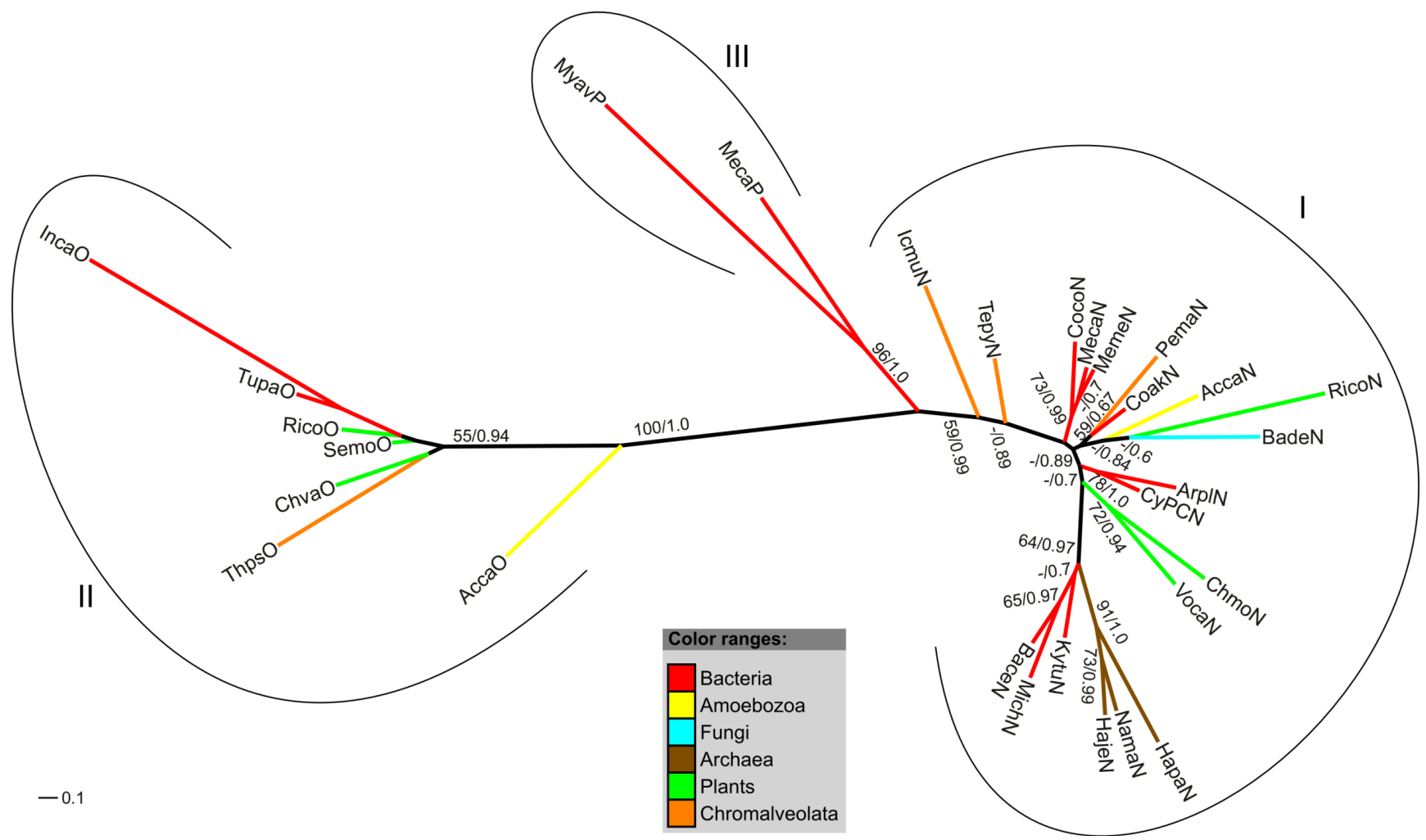

Figure 5. Radial maximum likelihood tree of trHb proteins. The colors of branches correspond to the taxonomic classification of the used sequences. Bootstrap support (bs) and posterior probability (PP) values equal or greater than $50 \%$ are given (bs/PP). The trHb proteins cluster in three highly supported clades (I-III), in accordance to their classification. For a description of used abbreviations please refer to Supplementary Material: table SI.

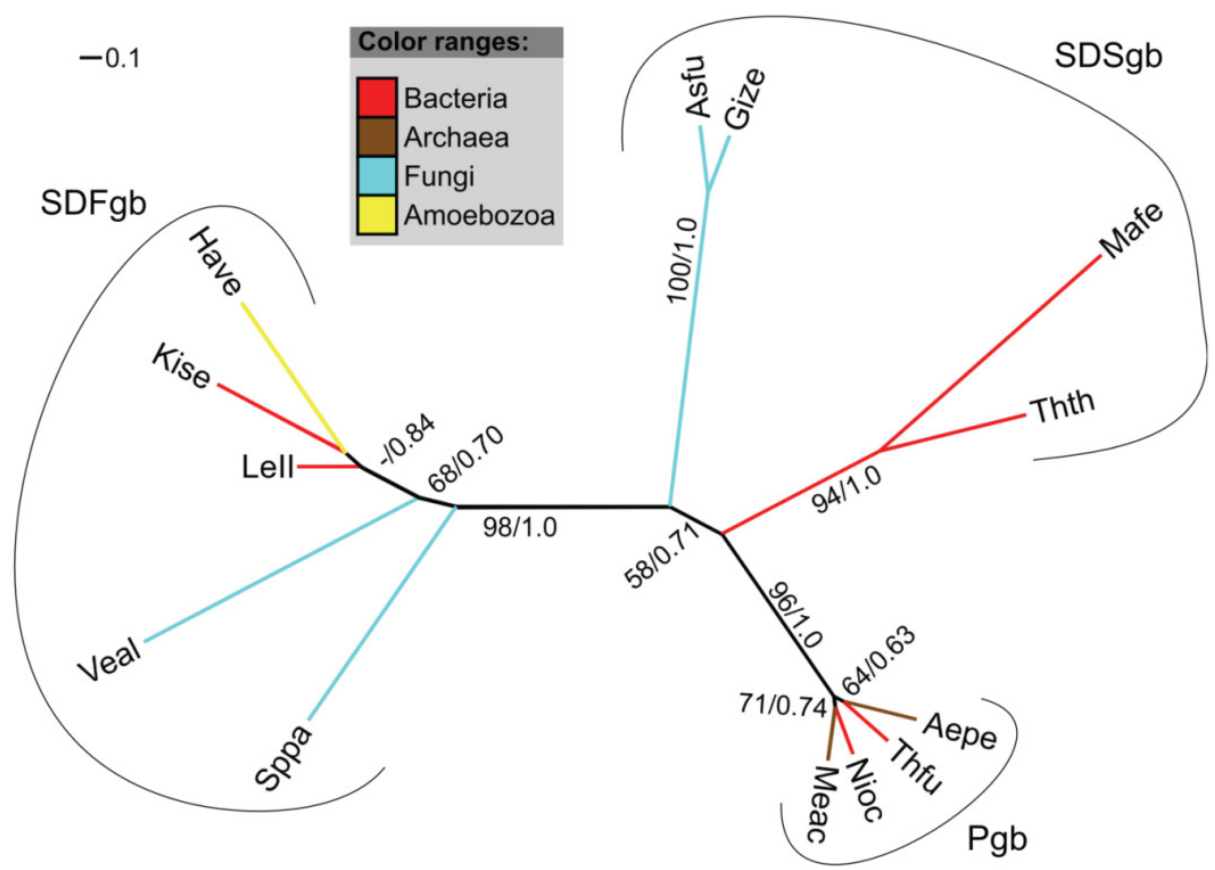

Figure 6. Radial maximum likelihood tree of single-domain globins and the SDFgb of $\boldsymbol{H}$. vermiformis. The colors of branches correspond to the taxonomic classification of the used sequences. Bootstrap support (bs) and posterior probability (pP) values equal or greater than $50 \%$ are indicated (bs/pp). The SDFgb of $H$. vermiformis (HaveSDFgb) clusters with two bacterial SDFgbs. For a description of used abbreviations please refer to Supplementary Material: table SI. 


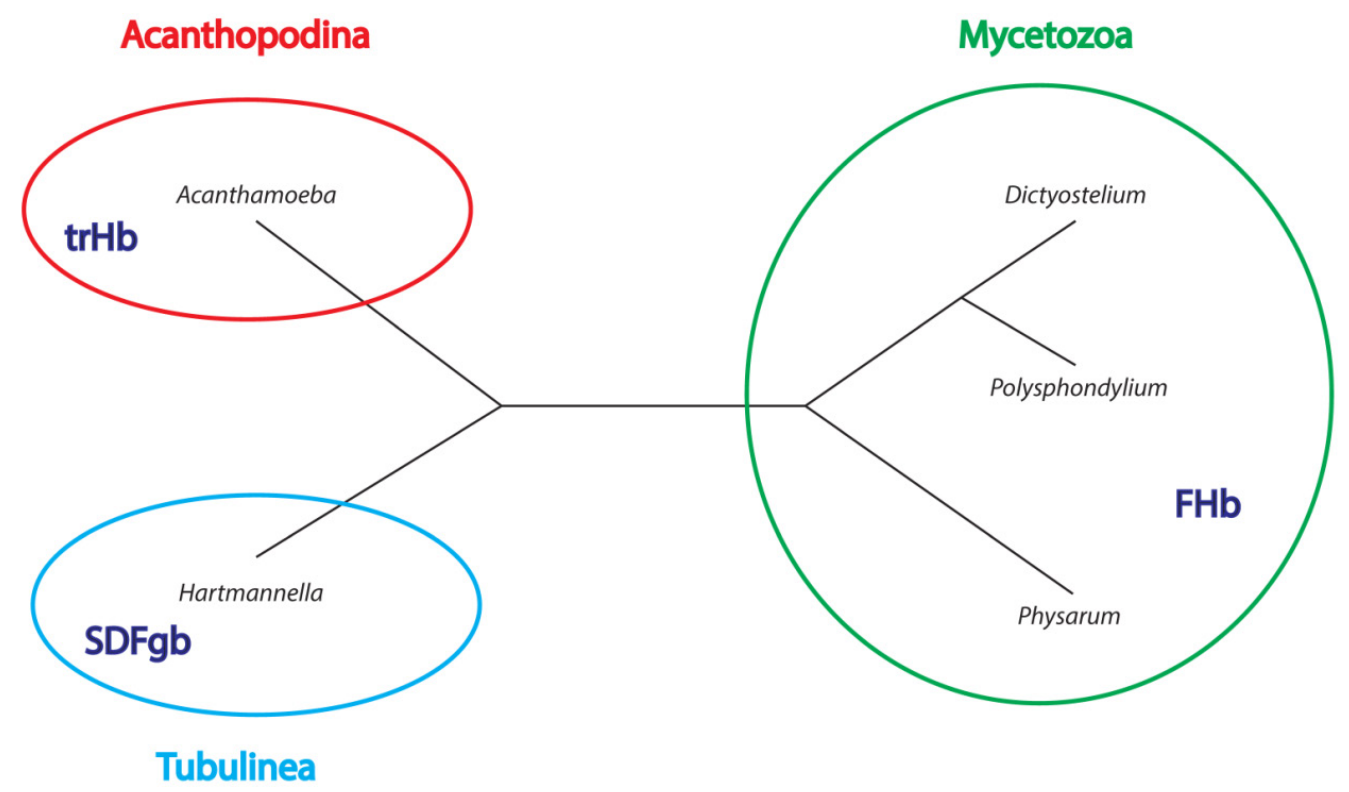

Figure 7. Phylegenetic relationships between studied Amoebozoa organisms. The tree is based on Adl et al. [9I] Please note that branch length is not to scale. Type of globin found in a given group is indicated in dark blue inside the group circles.

The phylogenetic tree of FHbs suggests that the three $\mathrm{FHb}$ genes of P. polycephalum (Phpo-1, Phpo-2, Phpo-3) arouse as a result of lineage-specific gene duplication events (Figure 3). Additionally, it can be inferred that the FHbs of D. fasciculatum and P. pallidum, as well as the FHbs of D. discoideum and D. purpureum share a common origin (Figure 7). The placement of AccaO in our phylogenetic trees is ambiguous and could be either basal to group II or to group I/III trHbs (Figure 5). Given its closer clustering to group II trHbs and the recognition of group II trHbs ahead of other trHbs in BLAST searches, we propose that AccaO represents a group II trHb.

Of the examined Amoebozoa species, H. vermiformis is the only one that possesses an SDFgb. Its closest relative is a globin of a Leptospirillum bacterium from the phylum Nitrospirae. The presence of an $\mathrm{SDFgb}$ in $\mathrm{H}$. vermiformis and absence in all other analyzed amoebozoan genomes could be explained by several independent losses in the other lineages. However, in light of its close relationship to bacterial SDFgbs, a horizontal gene transfer (HGT) event from an ancient bacterium seems to be more plausible. Moreover, increasing evidence suggests that HGT played a major role in the evolution of many species $[25,26]$ and it is assumed that HGT is an important force that shaped the phyletic distribution of globin genes [5-7, 18-21].

Likewise, the free-living amoeba $A$. castellanii is the only examined species that contains trHbs. In our phylogenetic trees (Figure 5) the trHbs cluster in accordance to their classification in three distinct groups that are highly supported. However, support for other clades is rather low and clustering of some proteins varies among the different methods. Therefore, it is difficult to draw precise conclusion from the phylogenetic analyses. However, the strong deviation of the inferred tree from the species tree and the absence of trHbs in the closely related genomes indicate a horizontal inheritance [79] of trHbs to an ancestor of the extant A. castellanii. Supportingly, several previous studies have already emphasized the importance of HGT of trHbs from prokaryotes to eukaryotes [16, 19-21]. Amoeba not only feed on bacteria, but can also harbor bacteria either as transient or stable endosymbionts [30, 80, 81]. The various interactions between free-living amoebae and bacteria may be the source of the horizontal transfer of an SDFgb and of trHbs to $H$. vermiformis and $A$. castellanii, respectively.

The $\mathrm{FHb}$ proteins of the social amoebas and the slime mold P. polycephalum were expected to be tightly related in view of the close relationship of the species, all belonging to the infraphylum Mycetozoa. However, in the inferred phylogenetic trees the FHbs groups are paraphyletic present in three distinct clades of which each contains some bacterial FHbs (Figure 3). Thus, it appears that the amoebozoan FHbs are closer related to some bacterial sequences than to their mycetozoan counterparts. One explanation of this tree would be that the common ancestor of Mycetozoa possessed multiple paralogous FHbs and that each of the described clades only retained one of them. However, given the previous thoughts, we favor a scenario in which the $\mathrm{FHb}$ genes were individually gained by HGT. In addition, two independent studies also observed the nesting of the FHbs from $D$. discoideum within a clade of some Firmicutes, $\beta$ - and $\varepsilon$-Proteobacteria and proposed the possibility of a 
prokaryote-to-eukaryote HGT event [6, 22]. It should be added that based on the tree presented in Figure 3, we cannot exclude possibility of the lateral transfer of the $\mathrm{FHb}$ gene in the other direction, i.e. from Eumycetozoa to bacteria (see clade 2). Similar observations were made for the $\mathrm{FHb}$ gene of the diplomonad Giardia lamblia [22] that in our tree (Gila) clusters in a common clade with the FHbs of D. fasciculatum (Difa) and P. pallidum (Popa). To further support such an evolutionary scenario, the likelihoods of the presented tree topology and of a topology in which the amoebozoan $\mathrm{FHb}$ cluster as a monophyletic group were compared, applying the AU test implemented in CONSEL [75, 77]. The monophyly of amoebozoan FHbs was rejected at a high confidence level ( $p$-value $3 \mathrm{e}^{-74}$ ), substantiating our assumption of three independent HGT events.

\section{Shared synteny among $D$. discoideum and $D$. purpureum}

The shared synteny among the FHbA genes of $D$. discoideum (DidiA) and D. purpureum (DipuA) supports an orthologous relationship of these two genes (Figure 1). In contrast, their orthology is not clearly evident from the phylogenetic tree (Figure 3). However, inference of phylogenetic trees is known to be error-prone, while shared synteny represents a reliable criterion for defining orthologs [82]. Apart from that, the phylogenetic tree (Figure 3) confirms the orthologous relationship of the $\mathrm{FHbB}$ genes (DidiB, DipuB) of these two Dictyostelium species. We hypothesize that the $\mathrm{FHbA}$ and $\mathrm{FHbB}$ genes emerged through the duplication of a pre- $\mathrm{FHb}$ gene in the common ancestor of D. discoideum and D. purpureum. This is supported by the retained linkage of the $\mathrm{FHb}$ genes in $D$. discoideum. Later the DipuB gene of D. purpureum got translocated to a new genomic location. Coincidently, Eichinger and colleagues observed that the genome of $D$. discoideum is enriched in relatively recently duplicated genes [83].

\section{Evolution of introns}

The presence or absence of introns and their positions vary widely among the analyzed globin genes. Although, D. discoideum and D. purpureum are closely related and possess highly conserved orthologous $\mathrm{FHb}$ genes, introns are not shared among the orthologs. While DidiB and DipuA harbor one and two introns, respectively, their orthologs are single exon genes (Figure 2). Thus, the introns have been either gained or lost after the divergence of $D$. discoideum and $D$. purpureum. The globin genes of almost all vertebrates, many invertebrates and several plants contain two introns at positions B12.2 and G7.0, which are considered as phylogenetically ancient $[1,84]$. None of the amoebozoan globin genes contains introns at these ancestral positions. Therefore, lineage-specific intron gains seem more likely than several intron loss events. The length of the introns is rather short and ranges from $74 \mathrm{bp}$ to $117 \mathrm{bp}$, with the exception of the intron in the $\mathrm{FHb}$ gene of $D$. fasciculatum. The genome analysis of for example $D$. discoideum and D. purpureum revealed only few and short introns with a mean length of 146 and $177 \mathrm{bp}$, respectively [83, 85]. Thus, the intron length of the globin genes does not deviate significantly from the overall intron length distribution in these species.

\section{Potential association of AccaN of A. castellanii with the membrane}

The in silico analysis of the trHbs of A. castellanii indicates that AccaN may be a membrane-bound globin protein. The presence of a potential transmembrane domain at its C-terminus was predicted by two independent tools, namely TMpred [53] and TMHMM [54]. Additionally, a possible palmitoylation site was found. Palmitoylation is a reversible lipid modification of proteins, which enhances the surface hydrophobicity and membrane affinity of proteins [86-88]. Although, palmitoylation occurs mainly close to the N-terminus of proteins, it has also been observed in other parts of proteins $[87,88]$. Globin proteins associated with the membrane have been identified in some bacteria [44-47], in the nematode Caenorhabditis elegans [89], in the shore crab Carcinus maenas [48], and recently also in vertebrates [49, 50]. However, there is no evidence that these globins share common ancestry suggesting that membrane-associated globins arouse independently several times in the course of evolution. A respiratory function of globins associated with the membrane is highly unlikely. It has been proposed that such bacterial globins facilitate oxygen transfer to the terminal oxidases of the respiratory chain [44, 46, 90]. Though eukaryotes lack a respiratory chain in their cell membranes, eukaryotic membrane associated globins may perform a comparable function, i.e. they may protect the membrane lipids from oxidative stress [48-50]. Alternatively, as suggested for vertebrate globin $\mathrm{X}$, AccaN may function as an $\mathrm{O}_{2}$ sensor or as a binding partner in a signal transduction pathway [49]. Any of these roles would be conceivable for AccaN and elucidating its function will also shed light upon its evolutionary history.

\section{Conclusion}

Globin genes are present in almost all eukaryotes, except for some unicellular parasites, such as Entamoeba histolytica and Plasmodium falciparum [5]. Although the globin genes of the social amoeba $D$. 
discoideum have been described and analyzed several years ago [34], nothing was known about the globin genes of the closely related species. This survey aimed to characterize the globin gene repertoire of amoebozoan species. Our results suggest lineage-specific adaptations of the globin gene repertoires; however presently there is no strong evidence of the adaptive processes in amoebozoan globin evolution and further studies are required to elaborate the issue. $\mathrm{FHb}$ genes were identified in several social amoebas and the true slime mold P. polycephalum of the infraphylum Mycetozoa, while trHb genes and an SDFgb gene were only found in A. castellanii (infraphylum Acanthopodina) and $H$. vermiformis (infraphylum Tubulinea), respectively. Intriguingly, the trHbN of $A$. castellanii might be associated with the membrane and thus may protect membrane lipids against oxidative stress.

Based on the phylogenetic analyses we propose that these globin genes are products of ancient HGTs from bacteria, though we cannot entirely rule out a scenario of ancient duplications and subsequent losses. These horizontal transfers could have been easily achieved given the tight interconnection between Amoebozoa and bacteria [80]. Nevertheless, our knowledge on the globin distribution in many other taxonomic groups of unicellular eukaryotes is still very limited. It would be captivating to examine the impact of horizontal gene transfer events on the globin gene diversity in these lineages.

\section{Supplementary Material}

Table S1: Table of sequences used in this study.

Table S2: Templates used for tertiary structure modeling employing Swiss-Model and SwissPDBViewer.

Figure S1: Bayesian tree of $\mathrm{trHb}$ proteins. The colors of branches correspond to the taxonomic classification of the used sequences. Posterior probability values equal or greater than $50 \%$ are indicated. The $\operatorname{trHb}$ proteins cluster in accordance to their classification in three distinct clades. For a description of used abbreviations please refer to table S1.

http://www.ijbs.com/v10p0689s1.pdf

\section{Acknowledgement}

This work was supported by the Institute of Bioinformatics funds and partially by the

FP7-People-2009-IRSES Project "EVOLGEN" No. 247633, the Asia-Africa S \& T Strategic Cooperation Promotion Program, and a Grant-in-Aid for Scientific Research on the Priority Area "Genome Science" from the Ministry of Education, Culture, Sports, Science and Technology of Japan. We are grateful to Marcin Jąkalski for his help in figure preparation.

\section{Author's contribution}

JD designed the study, carried out the bioinformatic analyses, and drafted the manuscript. DB conducted the transcriptome sequencing and assembly. YS conceived the transcriptome sequencing. WM conceived the study, participated in its design and coordination, and helped to draft the manuscript. All authors read and approved the final manuscript.

\section{Competing Interests}

The authors have declared that no competing interest exists.

\section{References}

1. Hardison RC: A brief history of hemoglobins: plant, animal, protist, and bacteria. Proc Natl Acad Sci U S A 1996, 93:5675-5679.

2. Freitas TA, Hou S, Dioum EM, Saito JA, Newhouse J, Gonzalez G, Gilles-Gonzalez MA, Alam M: Ancestral hemoglobins in Archaea. Proc Natl Acad Sci U S A 2004, 101:6675-6680.

3. Vinogradov S, Hoogewijs D, Vanfleteren J, Dewilde S, Moens L, Hankeln T: Evolution of the globin superfamily and its function. In Homoglobin: Recent Developments and Topics. Edited by Nagai M. Kerala, IND: Research Signpost; 2011: 231-254

4. Vinogradov SN, Moens L: Diversity of globin function: enzymatic, transport, storage, and sensing. The Journal of biological chemistry 2008, 283:8773-8777.

5. Vinogradov SN, Hoogewijs D, Bailly X, Arredondo-Peter R, Gough J, Dewilde S, Moens L, Vanfleteren JR: A phylogenomic profile of globins. BMC Evol Biol 2006, 6:31.

6. Vinogradov SN, Hoogewijs D, Bailly X, Arredondo-Peter R, Guertin M, Gough J, Dewilde S, Moens L, Vanfleteren JR: Three globin lineages belonging to two structural classes in genomes from the three kingdoms of life. Proc Natl Acad Sci U S A 2005, 102:11385-11389.

7. Vinogradov SN, Hoogewijs D, Bailly X, Mizuguchi K, Dewilde S, Moens L, Vanfleteren JR: A model of globin evolution. Gene 2007, 398:132-142.

8. Kendrew JC, Bodo G, Dintzis HM, Parrish RG, Wyckoff H, Phillips DC: A three-dimensional model of the myoglobin molecule obtained by $\mathrm{x}$-ray analysis. Nature 1958, 181:662-666.

9. Pesce A, Couture $\mathrm{M}$, Dewilde $\mathrm{S}$, Guertin $\mathrm{M}$, Yamauchi $\mathrm{K}$, Ascenzi P, Moens $\mathrm{L}$ Bolognesi M: A novel two-over-two alpha-helical sandwich fold is characteristic of the truncated hemoglobin family. The EMBO journal 2000, 19:2424-2434.

10. Hausladen A, Stamler JS: Is the flavohemoglobin a nitric oxide dioxygenase? Free radical biology \& medicine 2012, 53:1209-1210; author reply 1211-1202

11. Forrester MT, Foster MW: Protection from nitrosative stress: a central role for microbial flavohemoglobin. Free radical biology \& medicine 2012, 52:1620-1633.

12. Bonamore A, Boffi A: Flavohemoglobin: structure and reactivity. IUBMB life 2008, 60:19-28.

13. Frey AD, Kallio PT: Bacterial hemoglobins and flavohemoglobins: versatile proteins and their impact on microbiology and biotechnology. FEMS microbiology reviews 2003, 27:525-545.

14. Freitas TA, Hou S, Alam M: The diversity of globin-coupled sensors. FEBS letters 2003, 552:99-104.

15. Wittenberg JB, Bolognesi M, Wittenberg BA, Guertin M: Truncated hemoglobins: a new family of hemoglobins widely distributed in bacteria, unicellular eukaryotes, and plants. The Journal of biological chemistry 2002, 277:871-874.

16. Vuletich DA, Lecomte JT: A phylogenetic and structural analysis of truncated hemoglobins. Journal of molecular evolution 2006, 62:196-210.

17. Ascenzi P, Bolognesi M, Milani M, Guertin M, Visca P: Mycobacterial truncated hemoglobins: from genes to functions. Gene 2007, 398:42-51.

18. Vinogradov SN, Hoogewijs D, Arredondo-Peter R: What are the origins and phylogeny of plant hemoglobins? Communicative E integrative biology 2011, 4:443-445.

19. Vinogradov SN, Fernandez I, Hoogewijs D, Arredondo-Peter R: Phylogenetic relationships of 3/3 and 2/2 hemoglobins in Archaeplastida genomes to bacterial and other eukaryote hemoglobins. Molecular plant 2011, 4:42-58.

20. Vazquez-Limon C, Hoogewiis D, Vinogradov SN, Arredondo-Peter R: The evolution of land plant hemoglobins. Plant science : an international journal of experimental plant biology 2012, 191-192:71-81.

21. Hoogewijs D, Dewilde S, Vierstraete A, Moens L, Vinogradov SN: A phylogenetic analysis of the globins in fungi. PLoS One 2012, 7:e31856.

22. Andersson JO, Sjogren AM, Davis LA, Embley TM, Roger AJ: Phylogenetic analyses of diplomonad genes reveal frequent lateral gene transfers affecting eukaryotes. Current biology : $C B$ 2003, 13:94-104.

23. Watts RA, Hunt PW, Hvitved AN, Hargrove MS, Peacock WJ, Dennis ES: A hemoglobin from plants homologous to truncated hemoglobins of microorganisms. Proc Natl Acad Sci U S A 2001, 98:10119-10124.

24. Moens L, Vanfleteren J, Van de Peer Y, Peeters K, Kapp O, Czeluzniak J, Goodman M, Blaxter M, Vinogradov S: Globins in nonvertebrate species: dispersal by horizontal gene transfer and evolution of the structure-function relationships. Molecular biology and evolution 1996, 13:324-333.

25. Brown JR: Ancient horizontal gene transfer. Nature reviews Genetics 2003, 4:121-132.

26. Keeling PJ: Functional and ecological impacts of horizontal gene transfer in eukaryotes. Current opinion in genetics $\mathcal{E}$ development 2009, 19:613-619.

27. Vinogradov SN, Bailly X, Smith DR, Tinajero-Trejo M, Poole RK, Hoogewijs D: Microbial eukaryote globins. Advances in microbial physiology 2013, 63:391-446. 
28. Smirnov AV, Chao E, Nassonova ES, Cavalier-Smith T: A revised classification of naked lobose amoebae (Amoebozoa: lobosa). Protist 2011, 162:545-570.

29. Cavalier-Smith T: A revised six-kingdom system of life. Biological reviews of the Cambridge Philosophical Society 1998, 73:203-266.

30. Rodriguez-Zaragoza S: Ecology of free-living amoebae. Critical reviews in microbiology 1994, 20:225-241.

31. Kessin RH: Dictyostelium: Evolution, Cell Biology, and the Development of Multicellularity. Reissue edn. Cambrige, UK: Cambridge University Press; 2010.

32. Stanley SL, Jr.: Amoebiasis. Lancet 2003, 361:1025-1034.

33. Marciano-Cabral F, Cabral G: Acanthamoeba spp. as agents of disease in humans. Clinical microbiology reviews 2003, 16:273-307.

34. Iijima M, Shimizu H, Tanaka $Y$, Urushihara H: Identification and characterization of two flavohemoglobin genes in Dictyostelium discoideum. Cell structure and function 2000, 25:47-55.

35. Altschul SF, Gish W, Miller W, Myers EW, Lipman DJ: Basic local alignment search tool. J Mol Biol 1990, 215:403-410.

36. O'Brien EA, Koski LB, Zhang Y, Yang L, Wang E, Gray MW, Burger G, Lang BF: TBestDB: a taxonomically broad database of expressed sequence tags (ESTs). Nucleic acids research 2007, 35:D445-451.

37. Burge CB, Karlin S: Finding the genes in genomic DNA. Curr Opin Struct Biol 1998, 8:346-354.

38. Hebsgaard SM, Korning PG, Tolstrup N, Engelbrecht J, Rouze P, Brunak S: Splice site prediction in Arabidopsis thaliana pre-mRNA by combining local and global sequence information. Nucleic acids research 1996, 24:3439-3452

39. Brunak S, Engelbrecht J, Knudsen S: Prediction of human mRNA donor and acceptor sites from the DNA sequence. J Mol Biol 1991, 220:49-65.

40. Shi J, Blundell TL, Mizuguchi K: FUGUE: sequence-structure homology recognition using environment-specific substitution tables and structure-dependent gap penalties. J Mol Biol 2001, 310:243-257.

41. Schultz J, Milpetz F, Bork P, Ponting CP: SMART, a simple modular architecture research tool: identification of signaling domains. Proc Natl Acad Sci U S A 1998, 95:5857-5864.

42. Gaudet P, Fey P, Basu S, Bushmanova YA, Dodson R, Sheppard KA, Just EM, Kibbe WA, Chisholm RL: dictyBase update 2011: web 2.0 functionality and the initial steps towards a genome portal for the Amoebozoa. Nucleic acids research 2011, 39:D620-624.

43. Kreppel L, Fey P, Gaudet P, Just E, Kibbe WA, Chisholm RL, Kimmel AR: dictyBase: a new Dictyostelium discoideum genome database. Nucleic acids research 2004, 32:D332-333.

44. Liu C, He Y, Chang Z: Truncated hemoglobin o of Mycobacterium tuberculosis: the oligomeric state change and the interaction with membrane components. Biochemical and biophysical research communications 2004, 316:1163-1172.

45. Rinaldi AC, Bonamore A, Macone A, Boffi A, Bozzi A, Di Giulio A: Interaction of Vitreoscilla hemoglobin with membrane lipids. Biochemistry 2006, 45:4069-4076.

46. Ramandeep, Hwang KW, Raje M, Kim KJ, Stark BC, Dikshit KL, Webster DA: Vitreoscilla hemoglobin. Intracellular localization and binding to membranes. The Journal of biological chemistry 2001, 276:24781-24789.

47. Bonamore A, Farina A, Gattoni M, Schinina ME Bellelli A, Boffi A. Interaction with membrane lipids and heme ligand binding properties of Escherichia coli flavohemoglobin. Biochemistry 2003, 42:5792-5801.

48. Ertas B, Kiger L, Blank M, Marden MC, Burmester T: A membrane-bound hemoglobin from gills of the green shore crab Carcinus maenas. The Journal of biological chemistry 2011, 286:3185-3193.

49. Blank M, Wollberg J, Gerlach F, Reimann K, Roesner A, Hankeln T, Fago A, Weber RE, Burmester T: A membrane-bound vertebrate globin. PLoS One 2011, 6:e25292.

50. Blank M, Burmester T: Widespread occurrence of N-terminal acylation in animal globins and possible origin of respiratory globins from a membrane-bound ancestor. Molecular biology and evolution 2012, 29:3553-3561.

51. Bologna G, Yvon C, Duvaud S, Veuthey AL: N-Terminal myristoylation predictions by ensembles of neural networks. Proteomics 2004, 4:1626-1632.

52. Ren J, Wen L, Gao X, Jin C, Xue Y, Yao X: CSS-Palm 2.0: an updated software for palmitoylation sites prediction. Protein engineering, design \& selection : PEDS 2008, 21:639-644

53. Hofmann K, Stoffel W: TMbase - A database of membrane spanning proteins segments. Biol Chem Hoppe-Seyler 1993, 374:166.

54. Sonnhammer EL, von Heijne G, Krogh A: A hidden Markov model for predicting transmembrane helices in protein sequences. Proceedings International Conference on Intelligent Systems for Molecular Biology; ISMB International Conference on Intelligent Systems for Molecular Biology 1998, 6:175-182.

55. Arnold K, Bordoli L, Kopp J, Schwede T: The SWISS-MODEL workspace: a web-based environment for protein structure homology modelling. Bioinformatics 2006, 22:195-201.

56. Guex N, Peitsch MC: SWISS-MODEL and the Swiss-PdbViewer: an environment for comparative protein modeling. Electrophoresis 1997, 18:2714-2723.

57. Bordoli L, Kiefer F, Arnold K, Benkert P, Battey J, Schwede T: Protein structure homology modeling using SWISS-MODEL workspace. Nature protocols 2009, 4:1-13.

58. Campanella JJ, Bitincka L, Smalley J: MatGAT: an application that generates similarity/identity matrices using protein or DNA sequences. BMC bioinformatics 2003, 4:29.

59. Felsenstein J: PHYLIP - Phylogeny Inference Package (Version 3.2). Cladistics 1989, 5:164-166.

60. Edgar RC: MUSCLE: multiple sequence alignment with high accuracy and high throughput. Nucleic acids research 2004, 32:1792-1797.

61. Katoh K, Kuma K, Toh H, Miyata T: MAFFT version 5: improvement in accuracy of multiple sequence alignment. Nucleic acids research 2005, 33:511-518.

62. Katoh K, Misawa K, Kuma K, Miyata T: MAFFT: a novel method for rapid multiple sequence alignment based on fast Fourier transform. Nucleic acids research 2002, 30:3059-3066.

63. Papadopoulos JS, Agarwala R: COBALT: constraint-based alignment tool for multiple protein sequences. Bioinformatics 2007, 23:1073-1079.

64. Lassmann T, Sonnhammer EL: Automatic assessment of alignment quality. Nucleic acids research 2005, 33:7120-7128.

65. Stamatakis A, Hoover P, Rougemont J: A rapid bootstrap algorithm for the RAxML Web servers. Syst Biol 2008, 57:758-771.
66. Stamatakis A: RAxML-VI-HPC: maximum likelihood-based phylogenetic analyses with thousands of taxa and mixed models. Bioinformatics 2006, 22:2688-2690.

67. Ronquist F, Huelsenbeck JP: MrBayes 3: Bayesian phylogenetic inference under mixed models. Bioinformatics 2003, 19:1572-1574.

68. Huelsenbeck JP, Ronquist F: MRBAYES: Bayesian inference of phylogenetic trees. Bioinformatics 2001, 17:754-755.

69. Darriba D, Taboada GL, Doallo R, Posada D: ProtTest 3: fast selection of best-fit models of protein evolution. Bioinformatics 2011, 27:1164-1165.

70. Whelan S, Goldman N: A general empirical model of protein evolution derived from multiple protein families using a maximum-likelihood approach. Molecular biology and evolution 2001, 18:691-699.

71. [Internet] Rambaut A, Drummond AJ. Tracer v1.4. http:// beast.bio.ed.ac.uk/Tracer.

72. Miller MA, Pfeiffer W, Schwartz T: Creating the CIPRES Science Gateway for inference of large phylogenetic trees. Proceedings of the Gateway Computing Environments Workshop (GCE) 2010;:1-8.

73. Letunic I, Bork P: Interactive Tree Of Life (iTOL): an online tool for phylogenetic tree display and annotation. Bioinformatics 2007, 23:127-128.

74. Letunic I, Bork P: Interactive Tree Of Life v2: online annotation and display of phylogenetic trees made easy. Nucleic acids research 2011, 39:W475-478.

75. Shimodaira $\mathrm{H}$, Hasegawa M: CONSEL: for assessing the confidence of phylogenetic tree selection. Bioinformatics 2001, 17:1246-1247.

76. Schmidt HA, Strimmer K, Vingron M, von Haeseler A: TREE-PUZZLE: maximum likelihood phylogenetic analysis using quartets and parallel computing. Bioinformatics 2002, 18:502-504.

77. Shimodaira H: An approximately unbiased test of phylogenetic tree selection. Syst Biol 2002, 51:492-508

78. Ilari A, Bonamore A, Farina A, Johnson KA, Boffi A: The X-ray structure of ferric Escherichia coli flavohemoglobin reveals an unexpected geometry of the distal heme pocket. The Journal of biological chemistry 2002, 277:23725-23732.

79. Philippe H, Douady CJ: Horizontal gene transfer and phylogenetics. Current opinion in microbiology 2003, 6:498-505.

80. Horn M, Wagner M: Bacterial endosymbionts of free-living amoebae. The Journal of eukaryotic microbiology 2004, 51:509-514.

81. Schmitz-Esser S, Toenshoff ER, Haider S, Heinz E, Hoenninger VM, Wagner M, Horn M: Diversity of bacterial endosymbionts of environmental acanthamoeba isolates. Applied and environmental microbiology 2008, 74:5822-5831.

82. Droge J, Pande A, Englander EW, Makalowski W: Comparative genomics of neuroglobin reveals its early origins. PLoS One 2012, 7:e47972.

83. Eichinger L, Pachebat JA, Glockner G, Rajandream MA, Sucgang R, Berriman M, Song J, Olsen R, Szafranski K, Xu Q, et al: The genome of the social amoeba Dictyostelium discoideum. Nature 2005, 435:43-57.

84. Dixon B, Pohajdak B: Did the ancestral globin gene of plants and animals contain only two introns? Trends Biochem Sci 1992, 17:486-488.

85. Sucgang R, Kuo A, Tian X, Salerno W, Parikh A, Feasley CL, Dalin E, Tu H, Huang E, Barry K, et al: Comparative genomics of the social amoebae Dictyostelium discoideum and Dictyostelium purpureum. Genome biology 2011, 12:R20.

86. Resh MD: Fatty acylation of proteins: new insights into membrane targeting of myristoylated and palmitoylated proteins. Biochimica et biophysica acta 1999, 1451:1-16.

87. Nadolski MJ, Linder ME: Protein lipidation. The FEBS journal 2007, 274:5202-5210.

88. Linder ME, Deschenes RJ: Palmitoylation: policing protein stability and traffic. Nature reviews Molecular cell biology 2007, 8:74-84.

89. Tilleman L, De Henau S, Pauwels M, Nagy N, Pintelon I, Braeckman BP, De Wael K, Van Doorslaer S, Adriaensen D, Timmermans JP, et al: An N-myristoylated globin with a redox-sensing function that regulates the defecation cycle in Caenorhabditis elegans. PLoS One 2012, 7:e48768.

90. Pathania R, Navani NK, Rajamohan G, Dikshit KL: Mycobacterium tuberculosis hemoglobin $\mathrm{HbO}$ associates with membranes and stimulates cellular respiration of recombinant Escherichia coli. The Journal of biological chemistry 2002, 277:15293-15302.

91. Adl SM, Simpson AG, Farmer MA, Andersen RA, Anderson OR, Barta JR, Bowser SS, Brugerolle G, Fensome RA, Fredericq S, et al: The new higher level classification of eukaryotes with emphasis on the taxonomy of protists. The Journal of eukaryotic microbiology 2005, 52:399-451. 